\title{
RESISTANCE TO ALTERNARIA BROWN SPOT OF NEW CITRUS HYBRIDS ${ }^{1}$
}

\author{
KELLY APARECIDA FERNANDES DE CAMPOS², FERNANDO ALVES DE AZEVEDO³, \\ MARINÊS BASTIANEL ${ }^{3}$, MARIÂNGELA CRISTOFANI-YALY ${ }^{3}$
}

ABSTRACT- Alternaria brown spot (ABS) disease is caused by the fungus of Alternaria alternata f. $s p$. citri, which causes injury in leaves, branches and fruits of citrus. The action of the pathogen is directly related to the presence of toxin receptors in susceptible genotypes. The objective of this study was to characterize a population of citrus hybrids obtained from controlled crosses between Pêra de Abril sweet orange and the hybrid of Murcott tangor x Pêra sweet orange (TM x LP 163) for response to ABS through the in vitro inoculation of fungal spores in young detached leaves. The fungus was isolated from the lesions of Murcott tangor fruits that exhibited ABS symptoms. Two hundred thirty-five hybrids were evaluated, and 70 (30\%) showed different levels of disease symptoms on detached leaves after 72 hours of inoculation with the fungus, and $165(70 \%)$ were asymptomatic. The frequency of segregation observed (165R:70S) and high level of heritability $\left(h^{2}=0.91\right)$ suggest that few genes may be involved in controlling the inheritance of ABS resistance in citrus.

Index terms: Alternaria alternata, genetic breeding, Murcott tangor, Pêra de Abril, sweet orange.

\section{RESISTÊNCIA DE NOVOS HÍBRIDOS DE CITROS À MANCHA MARROM DE ALTERNARIA}

RESUMO - A mancha marrom de alternaria (MMA) é uma doença causada pelo fungo Alternaria alternataf. $s p$. citri, que causa lesões em frutos, folhas e ramos de citros. A ação do patógeno está diretamente relacionada à presença de receptores de toxina em genótipos suscetíveis. O objetivo deste estudo foi caracterizar uma população de híbridos de citros obtidos a partir de cruzamentos controlados entre a laranja Pêra de Abril e o híbrido de tangor Murcott x laranja Pêra (TM x LP 163) para resposta à MMA através da inoculação in vitro de esporos do fungo em folhas jovens destacadas. Um isolado do fungo foi obtido das lesões de frutos de tangor Murcott que exibiram sintomas de MMA. Duzentos e trinta e cinco híbridos foram avaliados, sendo que $70(30 \%)$ apresentaram níveis diferentes de sintomas de doença em folhas destacadas após 72 horas da inoculação com o fungo e $165(70 \%)$ foram assintomáticos. A frequência de segregação observada (165R:70S) e alta herdabilidade $\left(h^{2} g=0,91\right)$ sugerem que poucos genes podem estar envolvidos no controle da herança da resistência à MMA em citros.

Termos de indexação: Alternaria alternata, melhoramento genético, tangor Murcott, Pêra de Abril, laranja

\footnotetext{
${ }^{1}$ (Paper 101-16) Received August 09, 2016. Accepted June 05, 2017. Part of first author's master dissertation at the Programa de Pós-graduação em Produção Vegetal e Bioprocessos Associados, Universidade Federal de São Carlos, Araras-SP.

${ }^{2}$ Biologist, MSc, Centro APTA Citros Sylvio Moreira, Instituto Agronômico (IAC) Cordeirópolis-SP. E-mail: kellyapf@gmail.com ${ }^{3}$ Agronomist engineer, $\mathrm{PhD}$, Scientific researchers at the Centro APTA Citros Sylvio Moreira, Instituto Agronômico (IAC) Cordeirópolis-SP. E-mails: fernando@ccsm.br; mbastianel@ccsm.br; mariangela@ccsm.br
} 


\section{INTRODUCTION}

The main diseases that affect the Brazilian citrus industry include tristeza (RODRIGUES et al., 2014), citrus canker (GRAHAM et al., 2004), citrus variegated chlorosis (COLETTA FILHO et al., 2007), leprosis (BASTIANEL et al., 2010) and huanglongbing (greening) (COLETTA FILHO et al., 2004). With respect to mandarins specifically, we highlight alternaria brown spot (ABS), which is caused by Alternaria alternata f. sp. citri, that affects the main commercial varieties and their hybrids in the country, such as Ponkan mandarin (Citrus reticulata Blanco) and Murcott tangor (C. reticulata x Citrus sinensis Osbeck) (AZEVEDO et al., 2015).

ABS was first identified in Australia in Emperor mandarin (C. reticulata Blanco) around 1903, and the disease soon spread to other places in the world; it is now present in several countries, such as Turkey, Spain, Italy, South Africa, the USA, China, Brazil and Argentina (STUART et al., 2009; CUENCA et al., 2013, 2016; HUANG et al., 2015). In Brazil, the disease was recorded in Rio de Janeiro in 2001 (GOES et al., 2001) in Dancy mandarin, and it later spread to other states, such as Minas Gerais, Rio Grande do Sul and São Paulo (SPOSITO et al., 2003; STUART et al., 2009).

ABS is mainly disseminated through conidia (asexual spores) of the fungus, and the process of colonization involves the production of host-specific toxin (HST) (TSUGE et al., 2013). ABS symptoms occur in leaves, branches and fruits. The symptoms initially appear as small lesions that expand by the action of the toxin and cause intense defoliation during periods of increased infection (TIMMER et al., 2003). The toxin type ACT produced by A. alternata $f$. sp. citri is widely described in the literature and is specific to a particular host range (KOHMOTO et al., 1991; PEEVER et al., 1999), including mandarins and some hybrids.

In fine branches, small cortical lesions occur, with or without a chlorotic halo. In mature fruits, necrotic spots 1 to $10 \mathrm{~mm}$ in diameter occur, and the external quality of these fruits is severely reduced, depreciating them for consumption (TIMMER et al., 2003; TSUGE et al., 2013; BASSIMBA et al., 2014).

Currently, ABS constitutes the main disease of mandarins ( $C$. reticulata) and their hybrids; the main varieties planted in orchards in the state of São Paulo, including Ponkan mandarin and Murcott tangor, are highly susceptible (AZEVEDO et al., 2010). ABS incidence leads to reduced productivity (PRATES, 2004; AZEVEDO et al.,
2010; BASSANEZI et al., 2014) and, consequently, economic loss (CHUNG, 2012).

The resistance of citrus plants to ABS has been observed in species of citrus such as sweet orange (STUART et al., 2009), willow leaf mandarins and mandarins like Fremont, Thomas, Clementina and Cravo (REIS et al., 2007; SOUZA et al., 2009; PACHECO et al., 2012).

Few studies have investigated the origin of resistance and susceptibility of citrus genotypes to ABS. Susceptibility is likely simple and controlled by dominant inheritance (DALKILIC et al., 2005; GULSEN et al., 2010; CUENCA et al. 2013, 2016). Thus, the "A" allele would be dominant for susceptibility, and "a" would be recessive for disease resistance.

Studies conducted using populations of triploid hybrids (CUENCA et al., 2013) and diploid hybrids (CUENCA et al., 2016) confirmed these results, and the authors suggested that Murcott tangor and Orlando tangelo are both susceptible and heterozygous and that willow leaf mandarins $(C$. deliciosa Tenore) and clementines (C. clementina) are resistant and homozygous recessive.

The present study aimed to characterize ABS resistance in a population of 235 citrus hybrids from crosses between Pêra de Abril sweet orange and a hybrid of Murcott tangor x Pêra sweet orange (TM x LP163).

\section{MATERIAL AND METHODS}

A population of 235 hybrids was obtained in 2010 from controlled crossings between Pêra de Abril sweet orange (C. sinensis) and TM x LP 163. The latter is a hybrid between Murcott tangor (C. reticulata $\mathrm{x} C$. sinensis) $\mathrm{x}$ Pêra sweet orange (C. sinensis), produced in 2010 . The population was available for evaluation in the greenhouse at the Sylvio Moreira Citrus APTA Center of the Agronomic Institute (IAC).

Isolates of A. alternata $f$. sp. citri were obtained from injured tissues of Murcott tangor fruits collected from plants grown in the field at the Sylvio Moreira APTA Citrus Center of the Agronomic Institute (IAC). Leaf tissue with characteristic lesions was cut into small pieces with a scalpel. Surface disinfection was then performed by immersion in commercial ethyl alcohol $(70 \%)$ for 1 minute followed by immersion in $2 \%$ sodium hypochlorite for 2 minutes, after which the samples were transferred to Petri dishes containing potato dextrose agar medium ( $200 \mathrm{~g}$ potato, $20 \mathrm{~g}$ dextrose, $15 \mathrm{~g} \mathrm{~L}^{-1}$ agar). 
To prevent the growth of other fungal contaminants, fungicide with carbendazim $(640 \mathrm{mg}$ $\left.\mathrm{L}^{-1}\right)$ as the active ingredient was added. To avoid the growth of bacteria, $50 \mu \mathrm{g} \mathrm{mL} \mathrm{m}^{-1}$ tetracycline was added to the culture medium. The plates were maintained under a photoperiod of 12 hours and a temperature of $25^{\circ} \mathrm{C}$ (SASSERON, 2008).

After seven days, media composed of $30 \mathrm{~g}$ of calcium carbonate, $20 \mathrm{~g}$ of sucrose and $20 \mathrm{~g}$ of agar was prepared to a volume of $1 \mathrm{~L}$ and poured into Petri plates. Fifty 5-mm-wide discs with mycelial tissue growth were then transferred to this medium to induce sporulation. This material was maintained at $27^{\circ} \mathrm{C}$ for 5 days under a 12 -hour photoperiod. The identity of the isolates was confirmed via the optical microscopy of asexual structures.

For in vitro inoculation, young leaves were collected from all hybrids and their parents. The ABS-susceptible Dancy mandarin and Murcott tangor varieties and the ABS-resistant Fremont mandarin variety (AZEVEDO et al., 2010) served as control genotypes. Three leaves per inoculated plant were placed in a Petri dish with moistened filter paper and cotton, which were previously sterilized. The leaves were sprayed with $2 \mathrm{~mL}$ of a suspension containing the spores at a concentration of $10^{5}$ conidia $\mathrm{mL}^{-1}$ according to the methodology described by Peever et al. (1999) and Canihos et al. (1999). The experiment was conducted in a growth chamber at $27^{\circ} \mathrm{C}$ under a 12 -hour photoperiod. The experimental design was completely randomized with three replicates per genotype, and each replicate was represented by one Petri dish.

The evaluations of symptoms caused by the fungus were performed at 24, 48 and 72 hours after inoculation by observing the presence of typical symptoms of the disease. The determination of severity was assessed for a subset of three leaves per treatment using a diagrammatic scale including nine levels of severity in leaves expressed as the percentage of infected area $(0,0.3,3.5,8.0,15,34$, 61, 80, 90 and 97\%) (MARTELLI et al., 2016).

To better understand the disease progress over time, it was calculated the area under the disease progress curve (AUDPC). Based on the results, a representative histogram of the population in relation to ABS severity was constructed using the software R.

The severity of the disease data at the three times of evaluation was used to calculate the AUDPC as follows:

$$
\mathrm{AUDPC}=\Sigma[((\mathrm{y} 1+\mathrm{y} 2) / 2) *(\mathrm{t} 2-\mathrm{t} 1)]
$$

where y1 and y2 are two consecutive assessments carried out at times $\mathrm{t} 1$ and $\mathrm{t} 2$, respectively. This calculation is a statistical analysis that allows the verification of the behavior of the disease by the progress curve (BERGAMIN, 1995) from mathematical models used previously.

The AUDPC values were used to calculate genetic parameters such as variation (genetic and environmental) and heritability. For this, the program SELEGEN - REML/BLUP was used (RESENDE and SILVA, 2014).

\section{RESULTS AND DISCUTION}

Table 1 shows the severity values assessed using the diagrammatic scale and the AUDPC. In the first evaluation, 24 hours after the inoculation of detached leaves in vitro, leaf lesions were observed in 54 hybrids $(22.9 \%)$ and in varieties used as positive controls (Murcott tangor and Dancy mandarin), as expected (Figure 1). After 48 hours of inoculation, 54 hybrids were considered symptomatic, and 14 new hybrids showed symptoms typical of the disease. At this evaluation stage, a higher percentage of affected leaf area in the leaves where symptoms started was observed at 24 hours. Values ranging from $0.3 \%$ ( 24 hours) to $61 \%$ (48 hours) of the leaf area with symptoms were also observed. Forty-eight hours after inoculation, a $22.98 \%$ ( 24 hours) to $28.94 \%$ increase in the percentage of individuals showing symptoms was observed.

After 72 hours, an increase in the area of necrosis on the leaves in which symptoms began within 24 hours was observed, and the hybrid PAx163-3 showed maximum severity, as assessed by the diagrammatic scale (97\%). Throughout the population, $165(70 \%)$ asymptomatic individuals were observed, and $70(30 \%)$ other individuals showed different levels of symptoms (Figure 2). Studies on inheritance of ABS resistance in citrus were based on crosses between mandarins varieties (CUENCA et al., 2013) and between mandarin and sweet orange (CUENCA et al., 2016). These studies stated that the inheritance of ABS resistance in citrus is controlled by a single recessive allele (DALKILIC et al., 2005; GULSEN et al., 2010; CUENCA et al., 2013). Thus, the "A" allele would be dominant for susceptibility, and "a" would be recessive for disease resistance (CUENCA et al., 2013, 2016). Therefore, segregation is expected in progeny arising from crosses between resistant and heterozygous ABS-susceptible parents or even between two heterozygous ABS susceptible ones. In the present work, the parents Pêra de Abril sweet orange and 
the hybrid TM $x$ LP 163, were asymptomatic, 165 (70\%) asymptomatic individuals of the progeny were observed and $70(30 \%)$ individuals showed different levels of symptoms (Figure 2). Thus, the segregation observed in $\mathrm{F}_{1}$ plants contrasts with that reported before (DALKILIC et al., 2005; GULSEN et al., 2010; CUENCA et al., 2013, 2016) and suggested that two genes were involved in genetic control of this trait, rather than a single gene. Then, we suggested that due to a complete dominance for both gene pairs, only if both kinds of dominant alleles were present the susceptible phenotype appears. When one gene is homozygous recessive or both genes are homozygous recessive, the susceptible phenotype is hidden. Further studies should be conducted to elucidate the genetic mechanisms involved in sweet orange or in other varieties that do not respond to a HST produced by the pathogen.

High heritability $\left(h^{2}=0.91\right)$ and genotypic variability expressed by the genotypic variation coefficient $(C v g i=228.25)$ (Table 2) were observed in the present work, indicating that the selection of resistant plants in the progeny can be successful. Namely, the hybrids have adequate genetic variability for selection.

TABLE 1- Severity (\%) and the area under the disease progress curve (AUDPC) of alternaria brown spot evaluated after inoculation with $A$. alternataf. sp. citri in hybrids, parents and citrus varieties.

\begin{tabular}{|c|c|c|c|c|c|c|c|c|c|c|c|c|c|c|c|c|c|}
\hline \multirow[t]{2}{*}{ Genotype } & \multicolumn{6}{|c|}{ Hours after inoculation } & \multicolumn{2}{|c|}{ AUDPC } & \multirow[t]{2}{*}{ Genotype } & \multicolumn{6}{|c|}{ Hours after inoculation } & \multicolumn{2}{|c|}{ AUDP } \\
\hline & $\overline{24 h}$ & & $48 \mathrm{~h}$ & & $72 \mathrm{~h}$ & & & & & $24 \mathrm{~h}$ & & $48 \mathrm{~h}$ & & $72 h$ & & & \\
\hline Fremont & 0.0 & $\mathrm{aA}$ & 0.0 & $\mathrm{aA}$ & 0.0 & $\mathrm{aA}$ & 0.0 & $\mathrm{a}$ & PAx163 180 & 0.0 & $\mathrm{aA}$ & 0.0 & $\mathrm{aA}$ & 0.0 & $\mathrm{aA}$ & 0.0 & \\
\hline PAx1631 & 0.0 & $\mathrm{aA}$ & 0.0 & $\mathrm{aA}$ & 0.0 & $\mathrm{aA}$ & 0.0 & a & PAx163 184 & 0.0 & . & 0.0 & $\mathrm{~d} A \mathrm{~A}$ & 0.0 & $\mathrm{aA}$ & 0.0 & \\
\hline PAx16310 & 0.0 & $\mathrm{aA}$ & 0.0 & $\mathrm{aA}$ & 0.0 & $\mathrm{aA}$ & 0.0 & $\mathrm{a}$ & PAx163 185 & 0.0 & $\mathrm{aA}$ & 0.0 & $\mathrm{aA}$ & 0.0 & $\mathrm{aA}$ & 0.0 & \\
\hline PAx163100 & 0.0 & $\mathrm{aA}$ & 0.0 & $\mathrm{aA}$ & 0.0 & $\mathrm{aA}$ & 0.0 & $\mathrm{a}$ & PAx163186 & 0.0 & $\mathrm{aA}$ & 0.0 & $\mathrm{aA}$ & 0.0 & $\mathrm{aA}$ & 0.0 & \\
\hline Ax163 101 & 0.0 & $\mathrm{aA}$ & 0.0 & $\mathrm{aA}$ & 0.0 & $\mathrm{aA}$ & 0.0 & $\mathrm{a}$ & PAx163 188 & 0.0 & $\mathrm{a}$ & 0.0 & & 0.0 & $\mathrm{aA}$ & 0.0 & \\
\hline PAx163 102 & 0.0 & $\mathrm{aA}$ & 0.0 & $\mathrm{aA}$ & 0.0 & $\mathrm{aA}$ & 0.0 & $\mathrm{a}$ & PAx163 193 & 0.0 & $\mathrm{aA}$ & 0.0 & 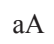 & 0.0 & $\mathrm{aA}$ & 0.0 & \\
\hline PAx163 104 & 0.0 & $\mathrm{aA}$ & 0.0 & $\mathrm{aA}$ & 0.0 & $\mathrm{aA}$ & 0.0 & $\mathrm{a}$ & PAx163 195 & 0.0 & $\mathrm{aA}$ & 0.0 & $\mathrm{aA}$ & 0.0 & $\mathrm{aA}$ & 0.0 & \\
\hline PAx163 108 & 0.0 & $\mathrm{aA}$ & 0.0 & $\mathrm{aA}$ & 0.0 & $\mathrm{aA}$ & 0.0 & $\mathrm{a}$ & PAx163 196 & 0.0 & $\mathrm{aA}$ & 0.0 & $\mathrm{aA}$ & 0.0 & $\mathrm{aA}$ & 0.0 & \\
\hline PAx163 11 & 0.0 & $\mathrm{aA}$ & 0.0 & $\mathrm{aA}$ & 0.0 & $\mathrm{aA}$ & 0.0 & $\mathrm{a}$ & PAx163 197 & 0.0 & $\mathrm{a} A$ & 0.0 & $\mathrm{aA}$ & 0.0 & $\mathrm{aA}$ & 0.0 & \\
\hline PAx163 110 & 0.0 & $\mathrm{aA}$ & 0.0 & $\mathrm{aA}$ & 0.0 & $\mathrm{aA}$ & 0.0 & $\mathrm{a}$ & PAx163 203 & 0.0 & $\mathrm{aA}$ & 0.0 & $\mathrm{aA}$ & 0.0 & $\mathrm{aA}$ & 0.0 & \\
\hline PAx163 112 & 0.0 & $\mathrm{aA}$ & 0.0 & $\mathrm{aA}$ & 0.0 & $\mathrm{aA}$ & 0.0 & $\mathrm{a}$ & PAx163 204 & 0.0 & $\mathrm{aA}$ & 0.0 & $\mathrm{aA}$ & 0.0 & $\mathrm{aA}$ & 0.0 & \\
\hline PAx163 113 & 0.0 & $\mathrm{aA}$ & 0.0 & $\mathrm{aA}$ & 0.0 & $\mathrm{aA}$ & 0.0 & $\mathrm{a}$ & PAx163 21 & 0.0 & $\mathrm{aA}$ & 0.0 & $\mathrm{aA}$ & 0.0 & $\mathrm{aA}$ & 0.0 & \\
\hline PAx163 117 & 0.0 & $\mathrm{aA}$ & 0.0 & $\mathrm{aA}$ & 0.0 & $\mathrm{aA}$ & 0.0 & $\mathrm{a}$ & PAx163 212 & 0.0 & $\mathrm{aA}$ & 0.0 & & 0.0 & $\mathrm{aA}$ & 0.0 & \\
\hline PAx163 119 & 0.0 & $\mathrm{aA}$ & 0.0 & $\mathrm{aA}$ & 0.0 & $\mathrm{aA}$ & 0.0 & $\mathrm{a}$ & PAx 1 & 0.0 & $\mathrm{aA}$ & 0.0 & $\mathrm{aA}$ & 0.0 & $\mathrm{aA}$ & 0.0 & \\
\hline PAx163 121 & 0.0 & $\mathrm{aA}$ & 0.0 & $\mathrm{aA}$ & 0.0 & $\mathrm{aA}$ & 0.0 & $\mathrm{a}$ & PAx163 215 & 0.0 & $\mathrm{aA}$ & 0.0 & $\mathrm{aA}$ & 0.0 & $\mathrm{aA}$ & 0.0 & \\
\hline PAx163 122 & 0.0 & $\mathrm{aA}$ & 0.0 & $\mathrm{aA}$ & 0.0 & $\mathrm{aA}$ & 0.0 & $\mathrm{a}$ & PAx163 220 & 0.0 & $\mathrm{a}$ & 0.0 & $\mathrm{aA}$ & 0.0 & $\mathrm{aA}$ & 0.0 & \\
\hline PAx 1 & .0 & $\mathrm{aA}$ & 0.0 & $\mathrm{aA}$ & 0.0 & $\mathrm{aA}$ & 0 & $\mathrm{a}$ & 22 & 0.0 & $\mathrm{a} A$ & 0.0 & & 0.0 & $\mathrm{aA}$ & 0.0 & \\
\hline PAx163 124 & .0 & $\mathrm{aA}$ & 0.0 & $\mathrm{aA}$ & 0.0 & $\mathrm{aA}$ & 0. & $\mathrm{a}$ & PAx163 223 & 0.0 & $\mathrm{a} A$ & 0.0 & & 0.0 & $\mathrm{aA}$ & 0.0 & \\
\hline PAx163 125 & 0.0 & $\mathrm{aA}$ & 0.0 & $\mathrm{aA}$ & 0.0 & $\mathrm{aA}$ & 0.0 & $\mathrm{a}$ & PAx163 224 & 0.0 & $\mathrm{aA}$ & 0.0 & $\mathrm{aA}$ & 0.0 & $\mathrm{aA}$ & 0.0 & \\
\hline PAx163 126 & 0.0 & $\mathrm{aA}$ & 0.0 & $\mathrm{aA}$ & 0.0 & $\mathrm{aA}$ & 0.0 & $\mathrm{a}$ & PAx163 226 & 0.0 & $\mathrm{aA}$ & 0.0 & $\mathrm{aA}$ & 0.0 & $\mathrm{aA}$ & 0.0 & \\
\hline PAx 1 & 0.0 & $\mathrm{aA}$ & 0.0 & $\mathrm{aA}$ & 0.0 & $\mathrm{aA}$ & 0.0 & $\mathrm{a}$ & PAx 1 & 0.0 & $\mathrm{aA}$ & 0.0 & & 0.0 & $\mathrm{aA}$ & 0.0 & \\
\hline PAx163 128 & 0.0 & $\mathrm{aA}$ & 0.0 & $\mathrm{aA}$ & 0.0 & $\mathrm{aA}$ & 0. & $\mathrm{a}$ & PAx 1 & 0.0 & $\mathrm{a}$ & 0.0 & $\mathrm{aA}$ & 0.0 & $\mathrm{aA}$ & 0.0 & \\
\hline PAx163 130 & 0.0 & $\mathrm{aA}$ & 0.0 & $\mathrm{aA}$ & 0.0 & $\mathrm{aA}$ & 0.0 & $\mathrm{a}$ & PAx163 236 & 0.0 & $\mathrm{aA}$ & 0.0 & $\mathrm{aA}$ & 0.0 & $\mathrm{aA}$ & 0.0 & \\
\hline 63132 & 0.0 & $\mathrm{aA}$ & 0.0 & $\mathrm{aA}$ & 0.0 & $\mathrm{aA}$ & 0.0 & $\mathrm{a}$ & PAx163 238 & 0.0 & $\mathrm{aA}$ & 0.0 & $\mathrm{aA}$ & 0.0 & $\mathrm{aA}$ & 0.0 & \\
\hline PAx163 133 & .0 & $\mathrm{aA}$ & 0.0 & $\mathrm{aA}$ & 0.0 & $\mathrm{aA}$ & & $\mathrm{a}$ & & & $\mathrm{a}$ & 0.0 & & 0.0 & $\mathrm{aA}$ & 0.0 & \\
\hline PAx163 134 & 0.0 & $\mathrm{aA}$ & 0.0 & $\mathrm{aA}$ & 0.0 & $\mathrm{aA}$ & 0.0 & $\mathrm{a}$ & PAx163 240 & 0.0 & $\mathrm{aA}$ & 0.0 & $\mathrm{aA}$ & 0.0 & $\mathrm{aA}$ & 0.0 & \\
\hline PAx163 138 & 0.0 & $\mathrm{aA}$ & 0.0 & $\mathrm{aA}$ & 0.0 & $\mathrm{aA}$ & 0.0 & $\mathrm{a}$ & PAx163 241 & 0.0 & $\mathrm{aA}$ & 0.0 & $\mathrm{aA}$ & 0.0 & $\mathrm{aA}$ & 0.0 & \\
\hline PAx163 140 & 0.0 & $\mathrm{aA}$ & 0.0 & $\mathrm{aA}$ & 0.0 & $\mathrm{aA}$ & 0 & $\mathrm{a}$ & PAx163 242 & 0.0 & $\mathrm{a}$ & 0.0 & & 0.0 & $\mathrm{aA}$ & 0.0 & \\
\hline PAx163 143 & 0.0 & $\mathrm{aA}$ & 0.0 & $\mathrm{aA}$ & 0.0 & $\mathrm{aA}$ & 0. & $\mathrm{a}$ & & 0.0 & $\mathrm{a}$ & 0.0 & & 0.0 & $\mathrm{aA}$ & 0.0 & \\
\hline PAx163 144 & 0.0 & $\mathrm{aA}$ & 0.0 & $\mathrm{aA}$ & 0.0 & $\mathrm{aA}$ & 0.0 & $\mathrm{a}$ & PAx163 244 & 0.0 & $\mathrm{aA}$ & 0.0 & $\mathrm{aA}$ & 0.0 & $\mathrm{aA}$ & 0.0 & \\
\hline PAx163 145 & 0.0 & $\mathrm{aA}$ & 0.0 & $\mathrm{aA}$ & 0.0 & $\mathrm{aA}$ & 0.0 & $\mathrm{a}$ & PAx163 247 & 0.0 & $\mathrm{aA}$ & 0.0 & $\mathrm{aA}$ & 0.0 & $\mathrm{aA}$ & 0.0 & \\
\hline PAx163 146 & 0.0 & $\mathrm{aA}$ & 0.0 & $\mathrm{aA}$ & 0.0 & $\mathrm{aA}$ & 0.0 & $\mathrm{a}$ & PAx163 248 & 0.0 & $\mathrm{aA}$ & 0.0 & $\mathrm{aA}$ & 0.0 & $\mathrm{aA}$ & 0.0 & \\
\hline PAx163 147 & 0.0 & $\mathrm{aA}$ & 0.0 & $\mathrm{aA}$ & 0.0 & $\mathrm{aA}$ & 0.0 & $\mathrm{a}$ & PAx 16325 & 0.0 & $\mathrm{aA}$ & 0.0 & $\mathrm{aA}$ & 0.0 & $\mathrm{aA}$ & 0.0 & \\
\hline PAx163 149 & 0.0 & $\mathrm{aA}$ & 0.0 & $\mathrm{aA}$ & 0.0 & $\mathrm{aA}$ & 0.0 & $\mathrm{a}$ & PAx163 250 & 0.0 & $\mathrm{aA}$ & 0.0 & $\mathrm{aA}$ & 0.0 & $\mathrm{aA}$ & 0.0 & \\
\hline PAx163 15 & 0.0 & $\mathrm{aA}$ & 0.0 & $\mathrm{aA}$ & 0.0 & $\mathrm{aA}$ & 0.0 & $\mathrm{a}$ & PAx163 251 & 0.0 & $\mathrm{aA}$ & 0.0 & $\mathrm{aA}$ & 0.0 & $\mathrm{aA}$ & 0.0 & \\
\hline PAx163 151 & 0.0 & $\mathrm{aA}$ & 0.0 & $\mathrm{aA}$ & 0.0 & $\mathrm{aA}$ & 0.0 & $\mathrm{a}$ & PAx163 252 & 0.0 & $\mathrm{aA}$ & 0.0 & $\mathrm{aA}$ & 0.0 & $\mathrm{aA}$ & 0.0 & \\
\hline PAx163 152 & 0.0 & $\mathrm{aA}$ & 0.0 & $\mathrm{aA}$ & 0.0 & $\mathrm{aA}$ & 0.0 & $\mathrm{a}$ & PAx163 253 & 0.0 & $\mathrm{aA}$ & 0.0 & $\mathrm{aA}$ & 0.0 & $\mathrm{aA}$ & 0.0 & \\
\hline PAx163 153 & 0.0 & $\mathrm{aA}$ & 0.0 & $\mathrm{aA}$ & 0.0 & $\mathrm{aA}$ & 0.0 & $\mathrm{a}$ & PAx163 254 & 0.0 & $\mathrm{aA}$ & 0.0 & $\mathrm{aA}$ & 0.0 & $\mathrm{aA}$ & 0.0 & \\
\hline PAx163 154 & 0.0 & $\mathrm{aA}$ & 0.0 & $\mathrm{aA}$ & 0.0 & $\mathrm{aA}$ & 0.0 & $\mathrm{a}$ & PAx163 255 & 0.0 & $\mathrm{aA}$ & 0.0 & $\mathrm{aA}$ & 0.0 & $\mathrm{aA}$ & 0.0 & \\
\hline
\end{tabular}


continuation...

$\begin{array}{llll}\text { PAx163 } 156 & 0.0 & \text { aA } & 0.0\end{array}$ $\begin{array}{llll}\text { PAx163 } 157 & 0.0 & \text { aA } & 0.0\end{array}$ $\begin{array}{llll}\text { PAx163 } 158 & 0.0 & \text { aA } & 0.0\end{array}$ PAx163 $159 \quad 0.0$ aA 0.0 $\begin{array}{lllll}P A x 163 & 160 & 0.0 & \text { aA } & 0.0\end{array}$ $\begin{array}{lllll}\text { PAx163 } 162 & 0.0 & \text { aA } & 0.0\end{array}$ $\begin{array}{lllll}P A x 163 & 164 & 0.0 & \text { aA } & 0.0\end{array}$ $\begin{array}{lllll}P A x 163 & 165 & 0.0 & \text { aA } & 0.0\end{array}$ $\begin{array}{lllll}\text { PAx163 } 166 & 0.0 & \text { aA } & 0.0\end{array}$ $\begin{array}{llll}\text { PAx163 } 167 & 0.0 & \text { aA } & 0.0\end{array}$ $\begin{array}{lllll}\text { PAx163 } 168 & 0.0 & \text { aA } & 0.0\end{array}$ $\begin{array}{lllll}\text { PAx163 } 169 & 0.0 & \text { aA } & 0.0\end{array}$ $\begin{array}{lllll}\text { PAx163 } 170 & 0.3 & \text { aA } & 0.0\end{array}$ $\begin{array}{lllll}\text { PAx163 } 171 & 0.0 & \text { aA } & 0.0\end{array}$

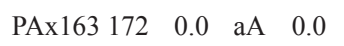
$\begin{array}{llll}\text { PAx163 } 173 & 0.0 & \text { aA } & 0.0\end{array}$

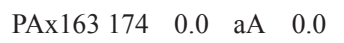
$\begin{array}{llll}\text { PAx163 } 175 & 0.0 & \text { aA } & 0.0\end{array}$ $\begin{array}{lllll}\text { PAx163 } 177 & 0.0 & \text { aA } & 0.0\end{array}$ $\begin{array}{llll}\text { PAx163 } 179 & 0.0 & \text { aA } & 0.0\end{array}$ $\begin{array}{lllll}\text { PAx163 } 33 & 0.0 & \text { aA } & 0.0\end{array}$ $\begin{array}{lllll}\text { PAx163 } 35 & 0.0 & \text { aA } & 0.0\end{array}$ $\begin{array}{lllll}\text { PAx163 } 38 & 0.0 & \text { aA } & 0.0\end{array}$ $\begin{array}{lllll}\text { PAx163 } 39 & 0.0 & \text { aA } & 0.0\end{array}$ $\begin{array}{llll}P A x 1634 & 0.0 & \text { aA } & 0.0\end{array}$ $\begin{array}{llll}P A x 16340 & 0.0 & \text { aA } & 0.0\end{array}$

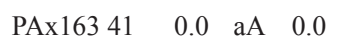

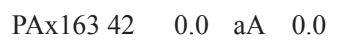
$\begin{array}{llll}\text { PAx163 } 44 & 0.0 & \text { aA } & 0.0\end{array}$

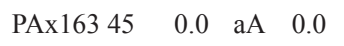
$\begin{array}{llll}\text { PAx163 } 46 & 0.0 & \text { aA } & 0.0\end{array}$ $\begin{array}{llll}\text { PAx163 } 47 & 0.0 & \text { aA } & 0.2\end{array}$ $\begin{array}{llll}\text { PAx163 } 48 & 0.0 & \text { aA } & 0.0\end{array}$ $\begin{array}{llll}\text { PAx163 } 49 & 0.0 & \text { aA } & 0.0\end{array}$ $\begin{array}{llll}\text { PAx163 } 5 & 0.0 & \text { aA } & 0.0\end{array}$ $\begin{array}{llll}\mathrm{PAx} 16350 & 0.0 & \mathrm{aA} & 0.0\end{array}$ $\begin{array}{llll}P A x 16351 & 0.0 & \text { aA } & 0.0\end{array}$ $\begin{array}{llll}\text { PAx163 } 52 & 0.0 & \text { aA } & 0.0\end{array}$ $\begin{array}{llll}P A x 16353 & 0.3 & \text { aA } & 0.3\end{array}$ $\begin{array}{llll}\text { PAx163 55 } & 0.0 & \text { aA } & 0.0\end{array}$ $\begin{array}{llll}\text { PAx163 } 57 & 0.0 & \text { aA } & 0.0\end{array}$ $\begin{array}{llll}\text { PAx163 } 6 & 0.0 & \text { aA } & 0.0\end{array}$ $\begin{array}{llll}P A x 16360 & 0.0 & \text { aA } & 0.0\end{array}$ $\begin{array}{llll}P A x 16361 & 0.0 & \text { aA } & 0.0\end{array}$ $\begin{array}{llll}\text { PAx163 63 } & 0.0 & \text { aA } & 0.0\end{array}$ $\begin{array}{lllll}\text { PAx163 64 } & 0.0 & \text { aA } & 0.0\end{array}$ $\begin{array}{lllll}P A x 16365 & 0.0 & \text { aA } & 0.0\end{array}$ $\begin{array}{llll}P A x 16366 & 0.0 & \text { aA } & 0.0\end{array}$ $\begin{array}{llll}P A x 16368 & 0.0 & \text { aA } & 0.0\end{array}$ $\begin{array}{llll}P A x 1637 & 0.0 & \text { aA } & 0.0\end{array}$ $\begin{array}{llll}P A x 16370 & 0.0 & \text { aA } & 0.0\end{array}$ $\begin{array}{llll}P A x 16371 & 0.0 & \text { aA } & 0.0\end{array}$ $\begin{array}{llll}P A x 16372 & 0.0 & \text { aA } & 0.0\end{array}$ $\begin{array}{llll}P A x 16375 & 0.0 & \text { aA } & 0.0\end{array}$ $\begin{array}{llll}P A x 16379 & 0.0 & \text { aA } & 0.0\end{array}$ $\begin{array}{llll}P A x 1638 & 0.0 & \text { aA } & 0.0\end{array}$ $\begin{array}{lllll}\mathrm{PAx} 16380 & 0.0 & \mathrm{aA} & 0.0\end{array}$ a 0.0 aA 0.0 aA 0.0 aA 0.0 aA 0.0 aA 0.0 aA 0.0 aA 0.0 aA 0.0 aA 0.0 aA 0.0 aA 0.0 aA 0.0 aA 0.0 aA 0.0 aA 0.0 aA 0.0 aA 0.0 aA 0.0 aA 0.0 aA 0.0 aA 0.0 aA 0.0 aA 0.0 aA 0.0 aA 0.0 aA 0.0 aA 0.0 aA 0.0 aA 0.0 aA 0.0 aA 0.2 aA 0.0 aA 0.0 aA 0.0 aA 0.0 aA 0.0 aA 0.0 aA 0.3 aA 0.0 aA 0.0 aA 0.0 aA 0.0 aA 0.0 aA 0.0 aA 0.0 aA 0.0 aA 0.0 aA 0.0 aA 0.0 aA 0.0 aA 0.0 aA 0.0 aA 0.0 aA 0.0 aA 0.0 aA 0.0
aA 0 aA $0.0 \quad$ a aA $0.0 \quad$ a $\mathrm{aA} \quad 0.0 \quad \mathrm{a}$ aA $0.0 \quad$ a aA $\quad 0.0 \quad$ a aA $\quad 0.0 \quad$ a aA $0.0 \quad$ a aA $0.0 \quad \mathrm{a}$ aA $0.0 \quad \mathrm{a}$ aA 0.0 aA 0.0 aA 0.0 aA 0.0 aA 0.0 aA 0.0 aA 0.0 aA 0.0 aA 0.0 aA 0.0 aA 0.0 aA 0.0 aA 0.0 aA 0.0 aA 0.0 aA 0.0 aA 0.0 aA 0.0 aA 0.0 aA 0.0 aA 0.0 aA 0.0 aA 0.0 aA 0.0 aA 0.0 aA 0.0 aA 0.0 aA 0.0 aA 0.0 aA 0.0 aA 0.0 aA 0.0 aA 0.0 aA 0.0 aA 0.0 aA 0.0 aA 0.0 aA 0.0 aA 0.0 aA 0.0 aA 0.0 aA 0.0 aA 0.0 aA 0.0 aA 0.0 aA 0.0 aA 0.0 PAx163 $258 \quad 0.0$ PAx163 $26 \quad 0.0$ PAx163 $260 \quad 0.0$ PAx163 $261 \quad 0.0$ PAx163 $263 \quad 0.0$ PAx163 $264 \quad 0.0$ PAx163 265 PAx163 266 PAx163 267 PAx163 269 PAx163 271 PAx163 273 PAx 163276 PAx163 277 PAx163 279 PAx163 28 PAx163 $280 \quad 0.0$ PAx163 $283 \quad 0.0$ PAx163 29 PAx 16378 PAx163 13 PAx163 218 PAx16393

PAx163 272

PAx163 275

PAx 16356

PAx163 111 PAx163 $69 \quad 0.0$ PAx163 $231 \quad 0.3$ PAx163 $105 \quad 3.9$ PAx163 $262 \quad 1.3$ PAx 16312 PAx163 $115 \quad 0.2$ PAx163 $141 \quad 3.9$ PAx163 249 PAx163 281 PAx163 62 PAx163 27 PAx163 $176 \quad 0.3$ PAx163 67 PAx 16376 PAx163 221 PAx163 31 PAx163 34 PAx163 58 PAx163 37 PAx163 217 PAx163 14 PAx163 198 PAx163 214 PAx163 74 PAx163 211 PAx163 109 PAx 16322 PAx163 23 PAx163 54
aA 0.0 aA $\quad 0.0 \quad \mathrm{aA}$ $\begin{array}{lll}\mathrm{aA} & 0.0 & \mathrm{aA}\end{array}$ $\begin{array}{lll}\mathrm{aA} & 0.0 & \mathrm{aA}\end{array}$ $\begin{array}{lll}\mathrm{aA} & 0.0 \quad \mathrm{aA}\end{array}$ $\begin{array}{lll}\mathrm{aA} & 0.0 \quad \mathrm{aA}\end{array}$ $\begin{array}{lll}\mathrm{aA} & 0.0 \quad \mathrm{aA}\end{array}$ $\begin{array}{lll}\mathrm{aA} & 0.0 \quad \mathrm{aA}\end{array}$ $\begin{array}{lll}\mathrm{aA} & 0.0 \quad \mathrm{aA}\end{array}$ $\begin{array}{lll}\mathrm{aA} & 0.0 \quad \mathrm{aA}\end{array}$ $\begin{array}{lll}\mathrm{aA} & 0.0 \quad \mathrm{aA}\end{array}$ $\begin{array}{lll}\mathrm{aA} & 0.0 & \mathrm{aA}\end{array}$ $\begin{array}{lll}\mathrm{aA} & 0.0 & \mathrm{aA}\end{array}$ aA 0.0 aA 0.0 aA 0.0 aA 0.0 aA 0.0 aA 0.0 aA 0.0 aA 0.3 aA 0.3 aA 0.3 aA 0.3 aA 1.4 aA $\quad 3.5$ aA 3.5 aA 3.5 aA 0.3 aA 3.5 bA 3.9 aA 3.8 aA 2.4 aA $\quad 3.9 \quad$ bB bA $\quad 3.9 \quad$ bA $\begin{array}{lll}\mathrm{aA} & 5.0 \quad \mathrm{cB}\end{array}$ aA $\quad 3.5 \quad b B$ aA $\quad 3.5 \quad b B$ bA $\quad 5.4 \quad \mathrm{cB}$ $\begin{array}{lll}\mathrm{aA} & 6.5 & \mathrm{cB}\end{array}$ $\begin{array}{lll}\mathrm{aA} & 0.3 \quad \mathrm{aA}\end{array}$ $\begin{array}{llll}\mathrm{aA} & 8.0 & \mathrm{~dB}\end{array}$ aA $10.3 \quad \mathrm{~dB}$ $\begin{array}{lll}\mathrm{aA} & 8.0 & \mathrm{~dB}\end{array}$ $\begin{array}{lll}\mathrm{aA} & 5.0 & \mathrm{cB}\end{array}$ $\begin{array}{llll}\mathrm{aA} & 5.0 & \mathrm{cB}\end{array}$ $\begin{array}{lll}\mathrm{aA} & 8.0 \quad \mathrm{~dB}\end{array}$ $\begin{array}{lll}\mathrm{aA} & 12.7 & \mathrm{eB}\end{array}$ bA 12.7 eB $\begin{array}{lll}\mathrm{aA} & 10.3 \quad \mathrm{~dB}\end{array}$ aA $\quad 15.0 \quad \mathrm{fB}$ aA 10.3 d aA $\quad 15.0 \quad \mathrm{fB}$ $\begin{array}{lll}\mathrm{aA} & 12.7 \quad \mathrm{eB}\end{array}$ $\begin{array}{lll}\mathrm{aA} & 12.7 & \mathrm{eB}\end{array}$ $\begin{array}{lll}\mathrm{aA} & 12.7 & \mathrm{eB}\end{array}$ aA 16.3 eB 
continuation...

\begin{tabular}{|c|c|c|c|c|c|c|c|c|c|c|c|c|c|c|c|}
\hline PAx163 81 & 0.0 & $\mathrm{aA}$ & 0.0 & $\mathrm{aA}$ & 0.0 & $\mathrm{aA}$ & 0.0 & $\mathrm{a}$ & PAx163 43 & 0.3 & $\mathrm{aA}$ & 15.0 & $\mathrm{fB}$ & $61.0 \mathrm{jC}$ & $1095.6 \mathrm{~g}$ \\
\hline PAx163 82 & 0.0 & $\mathrm{aA}$ & 0.0 & $\mathrm{aA}$ & 0.0 & $\mathrm{aA}$ & 0.0 & $\mathrm{a}$ & PAx163 259 & 1.4 & $\mathrm{aA}$ & 15.0 & $\mathrm{fB}$ & $61.0 \mathrm{jC}$ & $1108.4_{\mathrm{g}}^{\circ}$ \\
\hline PAx163 83 & 0.0 & $\mathrm{aA}$ & 0.0 & $\mathrm{aA}$ & 0.0 & $\mathrm{aA}$ & 0.0 & & PAx163 118 & 1.4 & $\mathrm{aA}$ & 12.7 & $\mathrm{eB}$ & $73.7 \mathrm{kC}$ & $1204.4_{\mathrm{g}}^{\circ}$ \\
\hline PAx163 84 & 0.0 & $\mathrm{aA}$ & 0.0 & $\mathrm{aA}$ & 0.0 & $\mathrm{aA}$ & 0.0 & & PAx163 199 & 0.3 & $\mathrm{aA}$ & 15.0 & $\mathrm{fB}$ & $80.0 \quad 1 \mathrm{C}$ & $1323.6_{\mathrm{h}}^{\circ}$ \\
\hline PAx163 85 & 0.0 & $\mathrm{aA}$ & 0.0 & $\mathrm{aA}$ & 0.0 & $\mathrm{aA}$ & 0.0 & $\mathrm{a}$ & PAx163 202 & 0.3 & $\mathrm{aA}$ & 15.0 & $\mathrm{fB}$ & $80.0 \quad 1 \mathrm{C}$ & $1323.6_{h}$ \\
\hline PAx16386 & 0.0 & $\mathrm{aA}$ & 0.0 & $\mathrm{aA}$ & 0.0 & $\mathrm{aA}$ & 0.0 & & PAx163 32 & 0.3 & $\mathrm{aA}$ & 15.0 & $\mathrm{fB}$ & $80.0 \quad 1 \mathrm{C}$ & $1323.6_{\mathrm{h}}$ \\
\hline PAx163 87 & 0.0 & $\mathrm{aA}$ & 0.0 & $\mathrm{aA}$ & 0.0 & $\mathrm{aA}$ & 0.0 & & PAx16399 & 0.3 & $\mathrm{aA}$ & 15.0 & $\mathrm{fB}$ & $80.0 \quad 1 C$ & $1323.6_{h}$ \\
\hline PAx163 88 & 0.0 & $\mathrm{aA}$ & 0.0 & $\mathrm{aA}$ & 0.0 & $\mathrm{aA}$ & 0.0 & & PAx163 229 & 1.2 & $\mathrm{aA}$ & 21.3 & $\mathrm{gB}$ & $70.7 \mathrm{mC}$ & $1374.0_{\mathrm{h}}$ \\
\hline PAx163 89 & 0.0 & $\mathrm{aA}$ & 0.0 & $\mathrm{aA}$ & 0.0 & $\mathrm{aA}$ & 0.0 & & PAx163 191 & 3.5 & $\mathrm{bA}$ & 21.3 & $\mathrm{gB}$ & $70.7 \mathrm{kC}$ & $1402.0_{\mathrm{h}}$ \\
\hline PAx16390 & 0.0 & $\mathrm{aA}$ & 0.0 & $\mathrm{aA}$ & 0.0 & $\mathrm{aA}$ & 0.0 & & PAx163 216 & 0.0 & $\mathrm{aA}$ & 15.0 & $\mathrm{fB}$ & $90.0 \mathrm{mC}$ & $1440.0_{\mathrm{h}}$ \\
\hline PAx16391 & 0.0 & $\mathrm{aA}$ & 0.0 & $\mathrm{aA}$ & 0.0 & $\mathrm{aA}$ & 0.0 & & PAx163120 & 0.3 & $\mathrm{aA}$ & 21.3 & $\mathrm{gB}$ & $83.31 \mathrm{C}$ & $1515.6 \mathrm{~h}$ \\
\hline PAx16394 & 0.0 & $\mathrm{aA}$ & 0.0 & $\mathrm{aA}$ & 0.0 & $\mathrm{aA}$ & 0.0 & & PAx163 19 & 5.4 & $\mathrm{bA}$ & 27.7 & $\mathrm{hB}$ & $73.7 \mathrm{kC}$ & $1613.2 \mathrm{i}$ \\
\hline PAx16395 & 0.0 & $\mathrm{aA}$ & 0.0 & $\mathrm{aA}$ & 0.0 & $\mathrm{aA}$ & 0.0 & & PAx163 268 & 0.3 & $\mathrm{aA}$ & 34.3 & $\mathrm{hB}$ & $67.3 \mathrm{jC}$ & $1635.2 \mathrm{~h}$ \\
\hline Pêra de Abril & 10.0 & $\mathrm{aA}$ & 0.0 & $\mathrm{aA}$ & 0.0 & $\mathrm{aA}$ & 0.0 & & PAx163 106 & 0.3 & $\mathrm{aA}$ & 27.7 & $\mathrm{hB}$ & $86.7 \mathrm{mC}$ & $1707.6_{i}$ \\
\hline TMxLP163 & 0.0 & $\mathrm{aA}$ & 0.0 & $\mathrm{aA}$ & 0.0 & $\mathrm{aA}$ & 0.0 & & PAx163 227 & 0.3 & $\mathrm{aA}$ & 27.7 & $\mathrm{hB}$ & $86.7 \mathrm{~m}$ & $1707.6 \mathrm{i}$ \\
\hline PAx163 237 & 0.0 & $\mathrm{aA}$ & 0.0 & $\mathrm{aA}$ & 0.3 & $\mathrm{aA}$ & 3.6 & & PAx1632 & 0.3 & $\mathrm{aA}$ & 34.0 & iB & $76.0 \mathrm{kC}$ & $1731.6_{\mathrm{i}}$ \\
\hline PAx163 103 & 0.1 & $\mathrm{aA}$ & 0.1 & $\mathrm{aA}$ & 0.1 & $\mathrm{aA}$ & 4.8 & & PAx163 131 & 3.5 & $\mathrm{bA}$ & 27.7 & $\mathrm{hB}$ & $86.7 \mathrm{mC}$ & $1746.0_{\mathrm{i}}$ \\
\hline PAx163 201 & 0.1 & $\mathrm{aA}$ & 0.1 & $\mathrm{aA}$ & 0.1 & $\mathrm{aA}$ & 4.8 & & Murcott & 3.5 & $\mathrm{bA}$ & 34.0 & iB & $83.31 \mathrm{C}$ & $1858.0_{j}$ \\
\hline PAx163 205 & 0.1 & $\mathrm{aA}$ & 1.4 & $\mathrm{aA}$ & 5.0 & $\mathrm{aA}$ & 9.4 & & PAx163 17 & 3.5 & $\mathrm{bA}$ & 34.0 & iB & $90.0 \mathrm{mC}$ & $1938.0_{j}$ \\
\hline PAx163 182 & 0.2 & $\mathrm{aA}$ & 0.2 & $\mathrm{aA}$ & 0.2 & $\mathrm{aA}$ & 9.6 & & PAx163 256 & 0.3 & $\mathrm{aA}$ & 36.7 & iB & $89.0 \mathrm{mC}$ & $1951.6_{j}^{J}$ \\
\hline PAx163 274 & 0.2 & $\mathrm{aA}$ & 0.3 & $\mathrm{aA}$ & 0.3 & $\mathrm{aA}$ & 13.2 & & PAx1633 & 0.3 & $\mathrm{aA}$ & 34.0 & iB & $97.0 \mathrm{mC}$ & $1983.6_{j}^{J}$ \\
\hline PAx163 129 & 0.3 & $\mathrm{aA}$ & 0.3 & $\mathrm{aA}$ & 0.3 & $\mathrm{aA}$ & 14.4 & 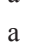 & Dancy & 5.0 & $\mathrm{bA}$ & 43.0 & jB & $86.7 \mathrm{mC}$ & $2132.0_{j}^{J}$ \\
\hline PAx163 207 & 0.3 & $\mathrm{aA}$ & 0.3 & $\mathrm{aA}$ & 0.3 & $\mathrm{aA}$ & 14.4 & a & PAx16398 & 0.3 & $\mathrm{aA}$ & 52.0 & $\mathrm{kB}$ & $94.7 \mathrm{mC}$ & $2387.6_{j}^{J}$ \\
\hline
\end{tabular}

$\begin{array}{lllllllll}\text { PAx163 } 230 & 0.3 & \text { aA } & 0.3 & \text { aA } & 0.3 & \text { aA } & 14.4 & \text { a }\end{array}$

${ }^{1}$ Means followed by the same letters in the column (lower) and in the line (capital) do not differ by the Scott-Knott test ( $\left.\mathrm{p}>0.05\right)$.

TABLE 2 - Estimated genetic parameters based on AUDPC values of $235 \mathrm{~F}_{1}$ hybrid progeny crossing between Pêra de Abril sweet orange vs TM x LP 163.

\begin{tabular}{cc}
\hline Genetic parameters & AUDPC \\
\hline$h^{2}$ & $0.91 \pm 0.10$ \\
$C V g i(\%)$ & 228.25 \\
$C V e(\%)$ & 70.88 \\
Mean & 215.86 \\
\hline
\end{tabular}

${ }^{1}$ Legend: $h^{2}{ }_{g}$ heritability of individual parcels in the broad sense, ie, the genotypic effects; CVgi (\%): genotypic coefficient of variation in percent; $\mathrm{CVe}$ (\%) environmental variation coefficient as a percentage; Mean: overall mean of the experiment. 


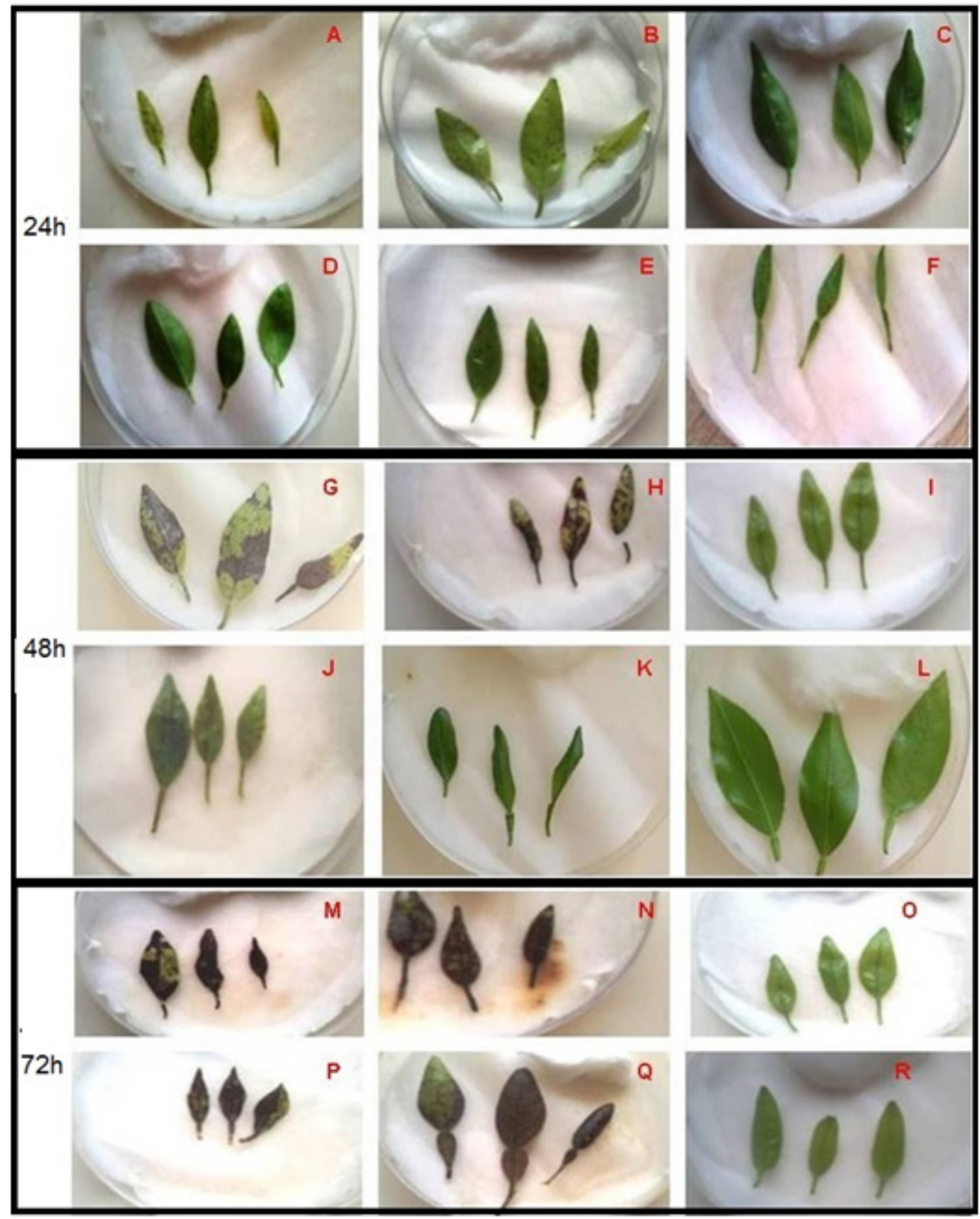

FIGURE 1- Leaves of hybrids showing typical lesions caused by A. alternata: $24 \mathrm{~h}$ after inoculation. A Dancy (Susceptible - S), B - Murcott (S), C - Fremont (Resistant - R), D - PAx163 85 (R), E PAx163 67 (S), F- PAx163 131 (S). 48 h after inoculation, G - Dancy, H - Murcott, I - Fremont, J - PAx163 37 (S), K - PAx163 69 (S) e L - PAx163 269 (R). 72 h after inoculation, M - Dancy, N - Murcott, O - Fremont, P - PAx163 67 (S), Q - PAx163 268 (S) and R - PAx163 80 (R). 


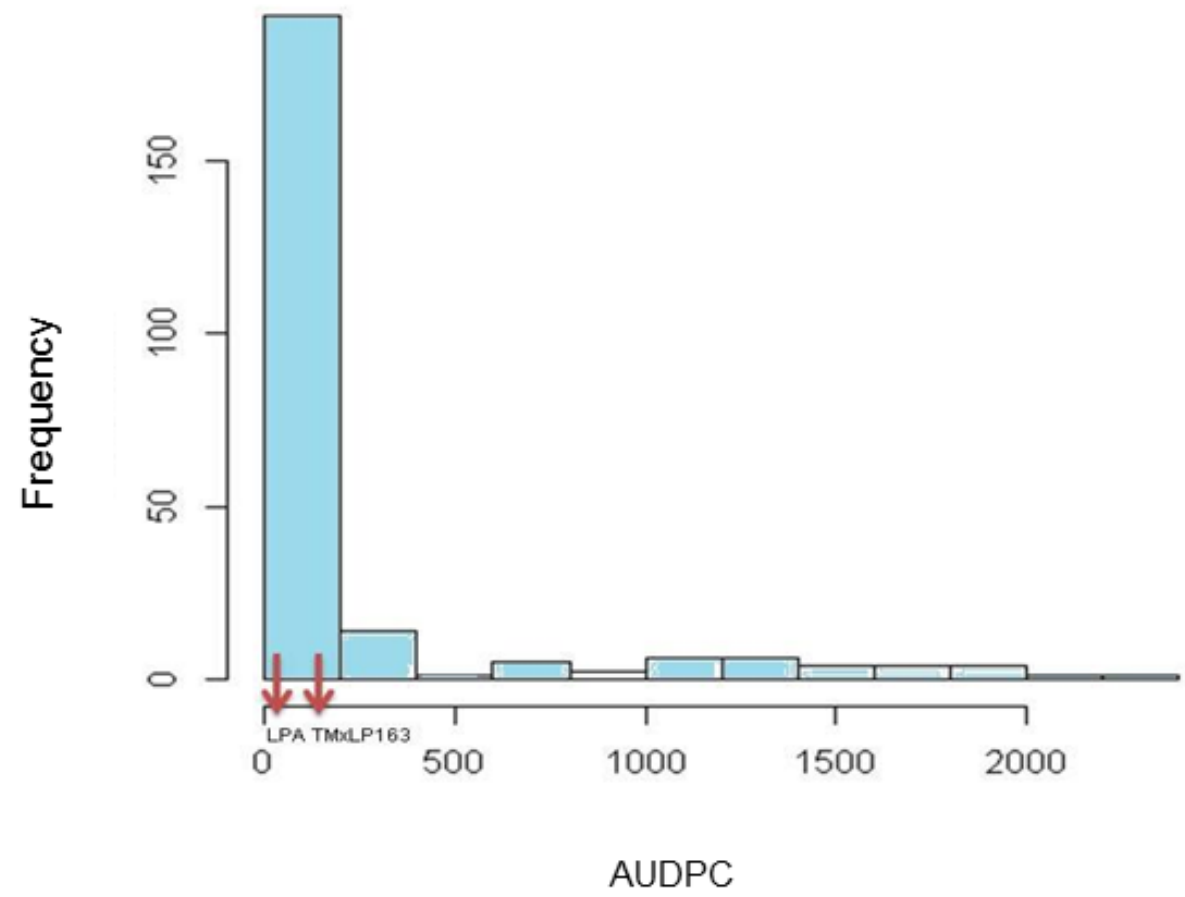

FIGURE 2- Histogram of frequency distribution of AUDPC means of 235 hybrids evaluated.

\section{CONCLUSION}

The frequency of segregation observed (165R:70S) and high heritability $\left(h^{2}=0.91\right)$ suggest that few genes may be involved in controlling the inheritance of ABS resistance in citrus.

\section{ACKNOWLEDGMENTS}

The authors thank the Conselho Nacional de Desenvolvimento Científico e Tecnológico (CNPq, proc. No 480426/2013-9), the Coordenação de Aperfeiçoamento de Pessoal de Nível Superior (CAPES) for scholarship granted and the Fundação de Amparo à Pesquisa do Estado de São Paulo (FAPESP - Proc. N N $^{\circ}$ 2011/18605-0, 2011/21559-0 and 2014/50880-0) for financial support.

\section{REFERENCES}

AZEVEDO,F.A.D.; MARTELLI,I.B.; POLYDORO, D. A.; PACHECO, C. D. A.; SCHINOR, E. H.; BASTIANEL, M. Positive relationship between citrus leaf miner and alternaria brown spot. Ciência Rural, Santa Maria, v. 45, n.7, 2015.
AZEVEDO, F.A.; POLYDORO, D.A.; BASTIANEL, M.; KUPPER, K.C.; STUART, R.M.; COSTA, F.P.; PIO, R.M. Resposta de diferentes genótipos de tangerinas e seus híbridos à inoculação in vitro e in vivo de Alternaria alternata. Revista Brasileira de Fruticultura, Jaboticabal, v. 32, n.1, p.1-10, 2010.

BASSANEZI, R.B.; AYRES, A.J.; MASSARI, C.A.; BELASQUE-JR, J.; BARBOSA, J.C. Progressão e distribuição espacial das principais pragas dos citros. In: ANDRADE, D.J.; FERREIRA, M. da C.; MARTINELLI, N.M. (Ed.). Aspectos da fitossanidade em citros. Jaboticabal: Cultura Acadêmica, 2014. p.31-50.

BASSIMBA, D.D.M.; MIRA, J.L.; VICENT, A. Inoculum sources, infection periods, and effects of environmental factors on Alternaria Brown Spot of mandarin in mediterranean climate conditions. Plant Disease, Saint Paul, v.98, n.3, p.409-417, 2014.

BASTIANEL, M.; NOVELLI, V.; KUBO, K.; KITAJIMA, E.W.; BASSANEZI, R.; MACHADO, M.A.; FREITAS-ASTÚA, J. Citrus Leprosis: centennial of na unusual mite-virus pathosystem. Plant Disease, Saint Paul, v.94, n.3, p.284-292, 2010. 
BERGAMIN, A.F. Curvas de progresso da doença. In: BERGAMIN FILHO, A.; KIMATI, H.; AMORIN, L. (Ed.). Manual de fitopatologia: princípios e conceitos. 3.ed. São Paulo: Agronômica Ceres, 1995. v.1, 602-625.

CANIHOS; PEEVER, T.L., TIMMER, L.W. Temperature, leaf wetness, and isolate effects on infection of Minneola tangelo leaves by Alternaria spp. Plant Disease, St Paul, v.83, p.429-433, 1999.

CHUNG, K-R. Stress response and pathogenicity of the necrotrophic fungal pathogen Alternaria alternata. Scientifica, London, v. 2012, p.1-17, 2012.

COLETTA-FILHO, H. D.; TAKITA, M.; TARGON, M.; CARLOS, E.; MACHADO, M. A bactéria Candidatus Liberibacter em plantas com huanglongbing (ex-greening) no Estado de São Paulo. Laranja, Cordeirópolis, v.25, n.2, p.367374, 2004.

COLETTA-FILHO, H.D.; PEREIRA, E.O.; SOUZA, A.A.; TAKITA, M.A.; CRISTOFANI-YALY, M.; MACHADO, M.A. Analysis of resistance to Xylella fastidiosa within a hybrid population of Pera sweet orange $\times$ Murcott tangor. Plant Pathology, Malden, 56, p. 661-668, 2007.

CUENCA, J.; ALEZA, P.; VICENT, A.; BRUNEL, D.; OLLITRAULT, P.; NAVARRO, L. Genetically based location from triploid populations and gene ontology of a 3.3-mb genome region linked to alternaria brown spot resistance in citrus reveal clusters of resistance genes. PloS One, San Francisco, v.8, n.10, p.e76755, 2013.

CUENCA, J.; ALEZA, P.; GARCIA-LOR, A.; OLLITRAULT, P.; NAVARRO, L. Fine Mapping for Identification of Citrus Alternaria Brown Spot Candidate Resistance Genes and Development of New SNP Markers for Marker-Assisted Selection. Fronties in Plant Science, 7:1948, 2016. doi: 10.3389/fpls.2016.01948.

DALKILIC, Z.; TIMMER, L.W.; GMITTER, F.G. Linkage of an Alternaria disease resistance gene in mandarin hybrids with RAPD fragments. Journal of the American Society for Horticultural Science, Alexandria, v.130, n. 2, p.191-195, 2005.
GOES, A.; MONTES DE OCA, A.G.; REIS, R.F. Ocurrencia de la mancha de alternaria em mandarina 'Dancy' en el Estado de Rio de Janeiro. Fitopatologia Brasileira, Botucatu, v.26, p.386, 2001.

GULSEN, O.; UZUN, A.; CANAN, I.; SEDAY, U.; CANIHOS, E. A new citrus linkage map based on SRAP, SSR, ISSR, POGP, RGA and RAPD markers. Euphytica, Dordrecht, v.173, n.2, p.265$277,2010$.

GRAHAM, J.H.; GOTTWALD, T.R.; CUBERO, J.; ACHOR, D.S. Xanthomonas axonopodis pv. citri: factors affecting successful eradication of citrus canker. Molecular Plant Pathology, Bristol, v. 5, n.1, p.1-15, 2004.

HIDALGO, M.R.; DE OLIVEIRA-MOLINA, R. Avaliação de cigarrinhas vetoras de Xylella fastidiosa no período da primavera na cidade de alto Paraná. SaBios-Revista de Saúde e Biologia, Campo Mourão, v.1, n.10, p.1-5, 2015.

HUANG, F.; FU, Y.; NIE, D.; STEWART, J. E.; PEEVER T.L.; LI, H. Identification of a novel phylogenetic lineage of Alternaria alternata causing citrus brown spot in China. Fungal Biology, Amsterdam, v.119, n.5, p.320-330, 2015.

KOHMOTO, K.; OTANI, H. Host recognition by toxigenic plant pathogens. Experientia, Basel, v. 47, n. 8, p. 755-764, 1991.

MARTELLI, I.B.; PACHECO, C.A.; BASTIANEL, M.; SCHINOR, E.H.; CONCEIÇÃO, P.M.; AZEVEDO, F.A. Diagrammatic scale for assessing foliar symptoms of alternaria brown spot in citrus. Agronomy Science and Biotechnology, v. 2, p. 56-61-61, 2016.

PACHECO, C.A.; MARTELLI, I.B.; POLYDORO, D.A.; SCHINOR, E.H.; PIO, R.M.; KUPPER, K.C., AZEVEDO, F.A. Resistance and susceptibility of mandarins and their hybrids to Alternaria alternata. Scientia Agricola, Piracicaba, v.69, n.6, p.386-392, 2012.

PEEVER, T.L.; CANIHOS, Y.; OLSEN, L.; IBAÑEZ, A.; LIU, Y.C.; TIMMER, L.W. Population genetic structure and host specificity of Alternaria spp. causing brown spot of Minneola tangelo and rough lemon in Florida. Phytopathology, St Paul, v.89, n.10, p.851-860, 1999. 
PRATES, H.S. Mancha de alternaria nas tangerinas. Revista Coopercitrus, Campinas, n.205, p.12-14, 2004.

REIS, R.F.; ALMEIDA, T.F.; STUCHI, E.S.; GOES, A. Susceptibility of citrus species to Alternaria alternata, the causal agent of the Alternaria brown spot. Scientia Horticulturae, Wageningen, v.113, p.336-342, 2007.

RESENDE, M.D.V.; SILVA, F.F. Estatística matemática, biométrica e computacional: modelos mistos e generalizados (REML/BLUP), inferência bayesiana, regressão aleatória, seleção genômica, qtl-gwas, estatística espacial e temporal, competição, sobrevivência. Viçosa: Universidade Federal de Viçosa/Departamento de Estatística, 2014.

RODRIGUES, A. S.; BARBOSA, C. D. J.; FILHO, W. D. S. S.; FREITAS-ASTÚA, J. Comportamento de híbridos de citros em relação à infecção natural pelo Citrus tristeza virus e à presença de sintomas de descamamento eruptivo. Revista Brasileira de Fruticultura, Jaboticabal - SP, v. 36, n. 3, p. 735741,2014

SASSERON, G. R. Desenvolvimento e validação de diagnóstico molecular de fungos patogênicos a citros. 2008. 71f. Dissertação (Mestrado em Genética, Melhoramento Vegetal e Biotecnologia) - Pós-Graduação - IAC.
SOUZA, M.C.; STUCHI, E.S.; GOES, A. Evaluation of tangerine hybrid resistance to Alternaria alternata. Scientia Horticulturae, New York, v.123, n.1, p.1$138,2009$.

SPÓSITO, M.B.; FEICHTENBERGER, E.; PIO, R.M.; CASTRO, J.L.; RENAUD, M. S.A. Ocorrência de mancha marrom de Alternaria em diferentes genótipos de citros nos estados de Minas Gerais, São Paulo e Rio Grande do Sul. Fitopatologia Brasileira, Brasília, DF, v.28, p.231, 2003.

STUART, R.M.; BASTIANEL, M. ; AZEVEDO, F.A ; MACHADO, M.A. . Alternaria brown spot. Laranja, Cordeirópolis, v.30, p.29-44, 2009.

TIMMER, L.W.; PEEVER, T.L.; SOLEIL, Z.; AZUYA, K.; KIMITSU, A. Alternaria diseases of citrus-novel pathosystems. Phytopathologia Mediterranea, Bologna, v.42, p.99-112, 2003.

TSUGE,T.; HARIMOTO, Y.; AKIMITSU, K.; OHTANI, K.; KODAMA, M.; AKAGI, Y.; EGUSA, M.; YAMAMOTO, M.; OTANI, H. Host-selective toxins produced by the plant pathogenic fungus Alternaria alternata. FEMS Microbiologia, Amsterdam, v.37, p.44-66, 2013. 